\title{
Burnout among paramedic students at a university in Johannesburg,
}

\section{South Africa}

\author{
C Stein, PhD; T Sibanda, BTech EMC
}

Department of Emergency Medical Care, Faculty of Health Sciences, University of Johannesburg, South Africa

Corresponding author: C Stein (cstein@uj.ac.za)

Background. Burnout has been studied in several emergency medical services contexts and has been found to be high compared with that found in other health professions. Although burnout among students has been described in several healthcare disciplines, this has not been done in the field of prehospital emergency care.

Objectives. To determine the prevalence of burnout among students in a 4-year university paramedic degree programme and to assess whether there was any significant difference in the prevalence of burnout among students during the 4 years of study.

Methods. In this cross-sectional survey all students enrolled in a 4-year university paramedic degree programme were invited to participate. The questionnaire consisted of 19 questions from the Copenhagen Burnout Inventory (CBI), combined with distractor questions. Responses were analysed descriptively and one-way analysis of variance was used to compare CBI scores across the 4 academic years of study.

Results. An $85 \%(n=93)$ response rate was obtained. The overall prevalence of burnout was $31 \%$. Mean CBI scores across all academic years of study were highest for personal burnout, followed by work-related burnout and patient care-related burnout. The highest prevalence of students with burnout was in the 4th year, as was the highest prevalence of work-related and personal burnout. The second highest prevalence of students with burnout was in the 1st year, as was the highest prevalence of patient care-related burnout. No significant difference was found in CBI total burnout scores across the 4 years of study.

Conclusion. Although there are no directly comparable data, the prevalence of burnout in this group of students appears to be high, particularly in the 1 st and 4 th years of study. Steps should be taken to ensure access to social and psychological support to avoid a negative impact on academic success and student wellbeing.

Afr J Health Professions Educ 2016;8(2):193-195. DOI:10.7196/AJHPE.2016.v8i2.626

Burnout, characterised by the well-described triad of emotional exhaustion, depersonalisation and a decreased sense of personal accomplishment, ${ }^{[1]}$ has been studied in a number of emergency medical services (EMSs) contexts. $^{[2-5]}$ The prevalence of burnout among paramedics varies, but is generally quite high compared with that in other health professions. In many cases stress from work environment-related factors appears to contribute to the development of burnout more than patient care-related factors. ${ }^{[2-5]}$

While qualified paramedics may draw on coping strategies developed over years of experience to mitigate the effects of work stress and subsequent development of burnout, students typically are not able to do this. Students also tend to face a more complex and multifactorial set of stressors, including stress related to the educational environment, the inherently stressful nature of clinical emergency care, and stress related to academic workload and success.

By considering Maslach's model of burnout and the typical academic life of medical students, Jennings ${ }^{[6]}$ argues that the relationship between academic success and burnout is bidirectional. The stress of academic workload and performance not only influences the development of burnout, but the latter may in turn negatively affect academic performance. ${ }^{[6-9]}$ Students therefore appear to have a more complex set of risk factors for the development of burnout and perhaps also have the most to lose when burnout does occur.

This study aimed to determine the prevalence of burnout among students in a 4-year paramedic university degree programme and to assess whether there was any significant difference in the prevalence of burnout among students during the 4 years of study.

\section{Methods}

We used a cross-sectional survey to assess the prevalence of burnout in this student population. The survey was conducted by using a directly administered questionnaire based on the Copenhagen Burnout Inventory (CBI). ${ }^{[10]}$ The CBI consists of 19 questions in three burnout categories: personal burnout (a state of prolonged physical and psychological exhaustion); work-related burnout (a state of prolonged physical and psychological exhaustion which is perceived as related to the participant's work or, in this case, academic activities); and patient-related burnout (a state of prolonged physical and psychological exhaustion which is perceived as related to the participant's work with patients) ${ }^{[10]}$ The term burnout was purposefully avoided anywhere in the questionnaire, i.e. the Student Wellness Questionnaire (SWQ). CBI questions were interspersed with a number of distractor questions.

The CBI questions use a Likert-type response scale and a system that assigns a score of between 0 and 100, depending on the response chosen. Once the inventory's scoring system had been applied to all responses, an average burnout score was calculated for each of the individual sections and for all three sections combined, giving a total burnout score, of which $\geq 50$ suggests a diagnosis of burnout. ${ }^{[10]}$ The validity, reliability and internal consistency of the CBI have been previously demonstrated. ${ }^{[10,11]}$

All students who were registered for the Emergency Medical Care (EMC) degree programme at the Department of Emergency Medical Care, University of Johannesburg, South Africa (SA) were invited to participate in the survey. The EMC programme spans 4 years of full-time study and is structured with a focus on basic sciences and diagnostic skills in the 
Table 1. Summary of academic structure for emergency medical care degree programme

\begin{tabular}{ll}
\hline Academic year of study & Main academic focus \\
\hline 1 & Basic sciences (anatomy, physiology, chemistry, physics), fundamental clinical content, limited clinical EMS exposure \\
2 & $\begin{array}{l}\text { Basic supporting sciences (diagnostics, pathology), clinical content, clinical exposure mostly in hospital (operating room, emergency } \\
\text { department, obstetrics) }\end{array}$ \\
3 & $\begin{array}{l}\text { Pharmacology, advanced adult clinical content, more intensive clinical EMS exposure } \\
\text { Specialised areas (paediatrics, thrombolysis, intensive care transfer), related clinical exposure (paediatrics, intensive care), ongoing } \\
\text { clinical EMS exposure, and a research project }\end{array}$ \\
EMS = emergency medical service. &
\end{tabular}

EMS = emergency medical service

Table 2. Descriptive data for CBI burnout scores $(n=93)$

\begin{tabular}{ll}
\hline Burnout score category & Mean $(\mathrm{SD})$ \\
\hline Work related & $49.1(12.9)$ \\
Personal & $53.4(15.0)$ \\
Patient care related & $34.0(19.5)$ \\
Total & $45.2(11.5)$
\end{tabular}

first 2 years, progressing to more advanced and specialised clinical practice in the last 2 years (Table 1).

Students were informed of the survey before or after scheduled lectures at the university campus. Those wishing to participate completed a consent form, followed by the SWQ. Data collection was completed between July and August 2012. All completed SWQs were collected and data were transcribed to an electronic spreadsheet application for further analysis. Transcribed data were checked for correctness.

CBI scores for each of the burnout subscales, and the total burnout score, were analysed descriptively. To establish whether a significant difference between CBI scores (subscale and total) existed across the 4 years of academic study, one-way analysis of variance (ANOVA) was used. The Statistical Package for the Social Sciences version 20 (SPSS 20) (IBM Corp., USA) was used and $p<0.05$ was considered significant for all statistical tests.

Ethical approval for the study was granted by the Faculty of Health Sciences Research Ethics Committee, University of Johannesburg (REC01-149-2015).

\section{Results}

Ninety-three $(85 \%)$ of a total of 110 students who registered for the EMC degree programme consented to participate in the survey. All SWQs handed out were completed and returned. Descriptive data for each of the burnout

Table 3. Descriptive data for CBI burnout scores across 4 years of study

\begin{tabular}{lllll}
\hline & \multicolumn{4}{l}{ CBI burnout scores, mean $(\mathrm{SD})$} \\
\cline { 2 - 5 } Burnout score category & 1st year & 2nd year & 3rd year & 4th year \\
\hline Work related & $50.10(12.92)$ & $44.86(10.75)$ & $48.76(14.70)$ & $53.40(13.01)$ \\
Personal & $52.53(14.50)$ & $52.81(16.46)$ & $55.06(16.0)$ & $55.80(14.17)$ \\
Patient care related & $38.63(21.93)$ & $25.00(15.81)$ & $33.82(17.55)$ & $34.33(16.35)$ \\
Total & $46.80(11.62)$ & $40.38(10.94)$ & $45.65(11.72)$ & $47.80(10.98)$
\end{tabular}

Table 4. Participants with CBI scores of $\geq 50$ ( $n=93)$

\begin{tabular}{lllll}
\hline \multirow{2}{*}{ Burnout score category } & \multicolumn{4}{c}{ CBI burnout scores of $\geq \mathbf{5 0 ,} \boldsymbol{n}(\%)$} \\
\cline { 2 - 5 } Work related & 1st year & 2nd year & 3rd year & 4th year \\
Personal & $24(60)$ & $7(33)$ & $11(65)$ & $10(67)$ \\
Patient care related & $24(60)$ & $14(67)$ & $10(59)$ & $10(67)$ \\
Total & $12(30)$ & $3(14)$ & $5(29)$ & $4(27)$ \\
& $14(35)$ & $5(24)$ & $3(18)$ & $7(47)$
\end{tabular}

categories and for total burnout scores across all academic years of study are given in Table 2 .

On average, students scored highest in the personal burnout category, with work-related burnout and patient care-related burnout categories yielding lower scores. The mean total burnout score was below the threshold value of 50 points. A total of 29 (31\%) students across all academic years had a total CBI score of $\geq 50$, the CBI threshold for burnout.

Descriptive data on burnout scores by academic year of study (Table 3) show that the mean work-related burnout score peaked in the 4th-year group, the mean personal burnout score was similar across all academic years of study, the mean patient care-related burnout score was highest in the 1st year, and the mean total burnout score was highest in the 4th year, followed closely by the 1st-year group.

Results obtained by selecting only students with CBI scores of $\geq 50$ in each burnout category are shown in Table 4. First-year students had the highest percentage of burnout scores of $\geq 50$ in the patient care-related burnout category, and slightly more than one-third of this group had total burnout scores of $\geq 50$. With the exception of the 2 ndyear group in the work-related burnout category, approximately two-thirds of students in each academic year had work-related and personal burnout scores of $\geq 50$.

Roughly one-third of students in each academic year had patient care-related burnout scores of $\geq 50$, with the exception of the 2nd-year group, where the prevalence was approximately half of this. The total burnout category showed the greatest variation, with the percentage of students having scores of $\geq 50$ ranging between $18 \%$ and almost three times this score. The greatest percentage of students with total burnout scores of $\geq 50$ was in 4 th year.

ANOVA results indicated that there was no significant difference in mean burnout scores between the 4 academic years of study in the categories of personal burnout $(p=0.866)$, work-related burnout ( $p=0.245)$, patient care-related burnout $(p=0.079)$, or total burnout $(p=0.456)$ scores. 


\section{Discussion}

No data currently exist on burnout prevalence among paramedic university students; therefore a direct comparison with a similar cohort is not possible. The $31 \%$ prevalence of total burnout, measured with the CBI, is very similar to that found among a group of qualified Advanced Life Support paramedics in Johannesburg (30\% prevalence) measured with the same instrument. ${ }^{[5]}$ It is higher than the prevalence of burnout identified in a number of other studies investigating this phenomenon among qualified paramedics. ${ }^{[2-4]}$

Burnout has been investigated and described in other healthcare student populations. The prevalence among medical students has been found to be comparatively high, with between $45 \%$ and $71 \%$ of students affected in different studies. ${ }^{[12-15]}$ Burnout among nursing students has been shown to be widely prevalent in one study, but at low to moderate levels, ${ }^{[16]}$ and has increased over the duration of educational contact in one population. ${ }^{[17]}$ The use of different scales to measure burnout makes direct comparison difficult, but it appears that the prevalence of burnout in this study was lower than that found in medical students but probably higher than that reported for nursing students. ${ }^{[12-15,16]}$

Our results suggest two main focal points of concern at extremes of the student academic experience - 1st year and 4th (final) year. Given the increased complexity and volume of academic work (including a research project), and clinical responsibility in the final year of the EMC programme, it is perhaps not surprising that the prevalence of work-related burnout, total burnout and personal burnout would be highest or among the highest in this group of students.

The 1st-year students' results were not anticipated, with this group having the highest prevalence of patient care-related burnout and the second highest total burnout prevalence. Although the clinical EMS exposure in the 1st year is not at an advanced level of care, as a first taste of the real world of EMS it may be rather overwhelming to these students, who have no prior experience. In a more general sense, 1st-year students may also find the transition from school to university life quite demanding and thus experience greater stress during their 1st year, giving rise to burnout.

The objective of this study did not include an assessment of the effect of burnout on academic performance. Studies investigating this relationship in other fields of study and academic environments have provided some evidence that the existence of burnout negatively affects academic performance and success mainly by a decrease in efficiency and productivity of students. ${ }^{[7-9]}$ It is therefore likely that a similar, albeit unquantified, effect may be present in this group of students.

The abovementioned burnout prevalence data and evidence linking these to a negative effect on academic performance highlight the need for available and effective social and psychological support referral pathways for EMC students, particularly those in 1st and 4th year. Even if such support is available and accessible, it could be argued that too much has already been lost in a fast-paced academic environment by the time a student or others recognise the need for referral.
Prevention of burnout can be attempted through various strategies, including person- and organisation-directed interventions, and a combination of both. The vast majority of such interventions have been found to be effective to some degree, with combined interventions having the best results over the longest time period. ${ }^{[18]}$ Ultimately, outcomes of even the most effective burnout prevention interventions will lessen over time, highlighting the importance of repeated preventive efforts. It is recommended that institutions offering EMC or similar academic programmes introduce ongoing burnout prevention interventions as one important way of contributing to the improved wellbeing of their students.

\section{Limitations of the study}

The sample used in this study contained larger student numbers in the 1st- and 2nd-year groups than in the 3rd- and 4th-year groups (40 and 21 v. 17 and 15 , respectively). The smaller 3rd- and 4th-year numbers may have increased the risk of a type 2 error in the ANOVA comparison of mean burnout scores across all academic years of study. The educational environment and circumstances at the University of Johannesburg, where students experience emergency care, cannot necessarily be compared with those of other universities or geographical areas; therefore the results of this study cannot necessarily be generalised compared with other EMC student populations in SA or elsewhere.

\section{References}

1. Schaufeli WB, Maslach C, Mazek T, eds. Professional Burnout: Recent Developments in Theory and Research. New York: Taylor and Francis, 1993.

2. Neale AV. Work stress in emergency medical technicians. J Occup Med 1991;33(9):991-997.

3. Chng CL, Eaddy S. Sensation seeking as it relates to burnout among emergency medical personnel: A Texas study. Prehosp Disaster Med 1999;14(4):36-40. DOI:10.1017/S1049023X00027709

Nirel N, Goldwag R, Feigenberg Z et al. Stress, work overload, burnout and satisfaction among paramedics in 4. Nirel N, Goldwag R, Feigenberg Z, et al. Stress, work overload, burnout and satisfation
Israel. Prehosp Disaster Med 2008;23(6):537-546. DOI:10.1017/S1049023X00006385

5. Stassen W, van Nugteren B, Stein C. Burnout among advanced life support paramedics in Johannesburg, South Africa. Emerg Med J 2013;30(4):331-334. DOI:10.1136/emermed-2011-200920]

Africa. Emerg Med J 2013;30(4):331-334. DOI:10.1136/emermed-2011-200920]
6. Jennings ML. Medical student burnout: Interdisciplinary exploration and analysis. J Med Humanit 6. Jennings ML. Medical student burnout: Interdis
2009;30(4):253-269. DOI:10.1007/s10912-009-9093-5

7. Galbraith CS, Merrill GB. Academic performance and burnout: An efficient frontier analysis of resource use efficiency among employed university students. J Further Higher Educ 2015;39(2):255-277. DOI:10.1080/030 9877X.2013.858673

8. Salanova M, Schaufeli W, Martinez I, Breso E. How obstacles and facilitators predict academic performance The mediating role of study burnout and engagement. Anxiety Stress Coping 2010;23(1):53-70. DOI: $10.1080 / 10615800802609965$

9. Yang $\mathrm{H}$. Factors affecting student burnout and academic achievement in multiple enrollment programs in Taiwan's technical-vocational colleges. Int J Educ Dev 2004:24(3):283-301. DOI:10.1016/j.jijedudev.2003.12.001

10. Kristensen TS, Borritz M, Villadsen E, et al. The Copenhagen Burnout Inventory: A new tool for the assessment of burnout. Work Stress 2005;19(3):192-207. DOI:10.1080/02678370500297720

11. Milfont TL, Denny S, Ameratunga S, et al. Burnout and wellbeing: Testing the Copenhagen Burnout Inventory in 1. Milfont TL, Denny S, Ameratunga S, et al. Burnout and wellbeing: Testing the Copenhagen B
New Zealand teachers. Soc Indic Res 2008;89(1):169-177. DOI:10.1007/s11205-007-9229-9

12. Dyrbye LN. Burnout and suicidal ideation among US medical students. Ann Intern Med 2008;149(5):334-341. DOI:10.7326/0003-4819-149-5-200809020-00008

13. Dyrbye LN, Massie SF, Eacker A, et al. Relationship between burnout and professional conduct and attitudes among US medical students. JAMA 2010;304(11):1173-1180. DOI:10.1001/jama.2010.1318

14. Guthrie E, Black D, Bagalkote H, et al. Psychological stress and burnout in medical students: A five year prospective longitudinal study. J R Soc Med 1998;91(5):237-243.

5. Dyrbye LN, Thomas MR, Eacker A, et al. Race, ethnicity, and medical student well-being in the United States Arch Intern Med 2007;167(19):2103-2109. DOI:10.1001/archinte.167.19.2103

16. Michalec B, Diefenbeck C, Mahoney M. The calm before the storm? Burnout and compassion fatigue among undergraduate nursing students. Nurse Educ Today 2013;33(4):314-320. DOI:10.1016/j.nedt.2013.01.026

7. Deary IJ, Watson R, Hogston R. A longitudinal cohort study of burnout and attrition in nursing students. J Adv Nurs 2003;43(1):71-81. DOI:10.1046/j.1365-2648.2003.02674.x

18. Awa WL, Plaumann M, Walter U. Burnout prevention: A review of intervention programs. Patient Educ Couns 2010;78(2):184-190. DOI:10.1016/.pec.2009.04.008 